Review

\title{
The Progress of BMP4 on the Role of Ocular Development and Eye Diseases
}

\author{
${ }^{1,2}$ Liang Jiaming, ${ }^{1}$ Wang Qian, ${ }^{2}$ Liu Xiaohe, ${ }^{1}$ Wang Shurong and ${ }^{1}$ Zhang Yan \\ ${ }^{I}$ Department of Ophthalmology, The 2nd Teaching Hospital of Jilin University, China \\ ${ }^{2}$ Norman Bethune Health Science of Jilin University, China
}

Article history

Received: 07-03-2017

Revised: 25-03-2017

Accepted: 05-04-2017

Corresponding Author:

Wang Shurong

Department of Ophthalmology,

The 2nd Teaching Hospital of

Jilin University, China

Email: srwang@jlu.edu.cn

\section{Zhang Yan}

Department of Ophthalmology, The 2nd Teaching Hospital of Jilin University, China

Email: zhangy66@jlu.edu.cn

Liang Jiaming and Wang Qian contributed equally to this work and should share the first authorship.

\section{Introduction}

Bone Morphogenetic, is an extracellular protein that plays a role in binding to cell surface proteins. BMP4 can inhibit the growth (Kallioniemi, 2012) of tumor cells and promote lung development (Lee et al., 2014). BMP4 can mediate apoptosis of retinal vascular endothelial cells and inhibit angiogenesis (Lynch et al., 2016). It has been found that BMP4 plays a vital role in the development of the eye (Boswell and Musil, 2015; Rubinstein et al., 2016; Huang et al., 2015). BMP4 is expressed in adult corneal epithelium, corneal cells and corneal endothelial cells. Also participated in the layered corneal epithelium cells, corneal epithelial cell chemotaxis and proliferation (Zhang et al., 2015; Sandri et al., 2016; Pereira da Silva et al., 2014). This article summarized the research progress of BMP4 in different tissues of the eye and briefly reviewed the role of BMP4 in different developmental stages.

\section{BMP 4 with Cornea, Sclera and Ciliary Body}

Ueno et al. (2016) studied the expression of BMP2, BMP4 and its receptor mRNA in human corneal epithelial cells, BMP2 and BMP4 of corneal fibroblast proliferation and apoptosis is also tested. It was found that BMP2 and BMP4 stimulate activation of NF- $\kappa \mathrm{B}$, while actinomycin D and SN50 peptides inhibit such activation and when activation of NF- $\mathrm{B}$ is inhibited, stimulation of the orneal fibroblasts by BMP2 and BMP4 leads to apoptosis of these cells. Thus, the expression of BMP2, BMP4 and its receptor in adult corneal cells was demonstrated. Keratinocyte proliferation and apoptosis can be regulated (Lee et al., 2014). It was found that the expression of BMP4 in keratoconus was higher by identifying differentially expressed genes between keratoconus and normal corneal cells (Allison et al., 2016). It is known that BMP4 mediates the apoptosis or alternative developmental fate of neural crest and other types of cells during development. Because the corneal cells originate from the neural crest, BMP4 stimulates the apoptosis of corneal fibroblasts and mediates the proliferation and apoptosis of corneal cells. The high expression of BMP4 in keratoconus indicated that BMP4 may play a vital role in the apoptosis of keratoconus.

The initial steps of iris development, BMP4 and BMP7 to regulate the expression of iris development at the right time and place, two genes by the tip cells in the optic cup neuroepithelium edge height expression. 
BMP2 and BMP4 are closely related to (Macé et al., 2011). Therefore, BMP4 may also promote the development of iris. In addition to their specific roles in the iris development, BMP4 also regulate the development of lens and ciliary epithelium. Therefore, it is suggested that some eye defects caused by BMP4 mutations may be due to abnormal iris, which provides a new direction for the treatment of some eye diseases.

Ciliary morphogenesis is a complex process, Chang (Jensen, 2005) found Bmp4 was expressed in mice ciliary bodies. In the $\mathrm{C} 57 \mathrm{BL} / 6 \mathrm{~J}$ background, Bmp4 heterozygous variable expression of anterior segment abnormalities, the most obvious features are elevated intraocular pressure and Shiram tube and the formation of ciliary body in some cases. Study on the subsequent Zhao (Napier and Kidson, 2007) has shown that the expression of Bmp4 plays an important role in the development of the ciliary body. Balenci et al. (2013; Zhang et al., 2013) found in adult stem cells in the human retina and the edge of the ciliary epithelium in rare retina can be in the absence of growth factor in the case of splitting all retinal cell types in vitro. BMP2, BMP4 and secreted coiled protein 2 are the major effectors of the antiproliferative effect of RSCs. Because of the similar induced quiescence in mice and human RSC in vitro, targeting these molecules can directly activate RSCs in the blind.

\section{BMP4 with Lens, Vitreous Body and Retina}

The development of vertebrate lens is a classical system to study the interaction of embryonic tissues. (Balenci et al., 2013) Found that Bmp4tm1 deficient mice were homozygous for the loss of lens and the loss of lens induced by. When cultured in vitro, exogenous BMP4 can be added to the optic vesicle. However, the use of BMP4 as an alternative to the explants in the culture of the optic vesicle does not lead to lens induction. BMP4 promotes the development of the lens, but it also needs to be regulated by the downstream genes, as a member of the multiplex signal.

Chang (Huang et al., 2015) found that Bmp4 deficiency $($ Bmp4 + ) haploid mice eye analysis showed that in most eyes examined in the vitreous body and dense vitreous body in irregular white spots, retinal vasculature in Bmp4 $+/$ - eyes is abnormal. Fluorescein angiography showed the presence of leakage in the vitreous body fluorescein small vascular tortuous dense network of vitreous body vascular anomalies lead to the lens and retina adhesion aggravate retinal detachment, thus obtains the BMP4 on the development of normal lens plays an important role.

Maruyama et al. (2006) Investigated the expression of BMP4 in the developing retina (Mao et al., 2015). In E19, a very strong BMP4 Immunoreactivity (IR) was found in the nerve fiber layer. In P1, the inner plexiform layer exhibits a very strong BMP4-IR. Since then, a large number of BMP4 expression remained in adulthood. These results suggest that BMP4 plays a key role in the retinal development process and in the grown man population. (Maruyama et al., 2006) Also evaluated the role of BMP4 signaling in the development of neural retina and Retinal Pigment Epithelium (RPE) by ectopic Bmp4 expression in the whole embryo culture system of mouse. The neural retina showed significant down regulation of specific markers, such as Vsx2 (Chx10) and Pax6. In RPE, pigmentation is affected in a phase dependent manner. When the eye tissue in the visible pigmentation stage ( (SS) 30-32 somite stage) before exposure to BMP4, the development of pigmentation is suppressed and when the pigmentation after the start $(\sim 34-35 \mathrm{ss})$ exposure, RPE showed reduced pigmentation. Further evaluation of ocular tissues revealed early genes (such as in the retina of Rx, Six 3 and BMP4) treatment in the eyes of RPE in Wnt13, Otx2, Mitf and other downstream pigmentation gene expression and BSA treated eyes compared to the significant changes that BMP4 changes in early gene expression and potential role in RPE and in the development of retina.

\section{BMP4 and Early Embryonic Eye Development}

The expression of BMPs in vertebrates, suggests an important features of these molecules in the eye during the development of the BMP4, which plays an important role in Trousse (Huang et al., 2015) using chick embryo as a model system, through the optic vesicles in the local culture proved BMP4 adjust the back of optic cup cell death. Takuma (Müller et al., 2007) in the study clearly shows that BMP4 constitute the dorsal ventral signal system and control the overall development of the eye.

\section{BMP4 and Ocular Diseases}

\section{BMP4 and Proliferative Vitreoretinopathy}

Hyperplastic vitreous body retina (PVR) Retinal Detachment (RD) is a serious complication of vision, which is characterized in that the formation of shrinkage fibrosis membrane, RPE Epithelial Mesenchymal Transition (EMT). Kobayashi et al. (2010) Exogenous BMP4 inhibits TGF-beta down regulation of epithelial markers and up regulation of mRNA and protein levels in PRE cells. Endogenous BMP4 stimulates changes in EMT markers. These results demonstrated that BMP4 may inhibit TGF-beta EMT of PRE cells and their shrinkage, suggesting a potential target for treatment of PVR.

\section{BMP4 and Glaucoma}

Glaucoma is usually accompanied by High Intraocular Pressure (IOP), which is caused by obstruction of intraocular fluid drainage. In some families, the abnormal development of the anterior segment contributes to glaucoma. In most of these families cause anterior hypoplasia and glaucoma genes have not been identified, understand the development process and affected little 
BMPs involved in various developmental processes, such as Chang (Yao et al., 2016) tested the importance of development and developmental glaucoma gene dosage on Bmp4. The results showed that Bmp4 $+/$ - mice does not exist or obstruction of the trabecular meshwork and Schlemm's canal drainage structure. With serious drainage the abnormal structure of more than $80 \%$ or more mice with elevated angle range of IOP, which proves that BMP4 heterozygous defects lead to anterior dysplasia and elevated IOP, these abnormalities and development of glaucoma in patients with abnormal human similarity. Therefore, BMP4 is a associated with glaucoma and other developmental conditions.

A unique gene was developed from the Primary Open-Angle Glaucoma (POAG), which developed the genes forkhead box $\mathrm{Cl}$ (FOXC1), transforming growth factor beta2 (TGF beta2) and BMP4. But the results show that the above gene in the pathogenesis of POAG has no special role (Ito and Walter, 2014), which also provides a new direction for the treatment of disease.

\section{BMP4 and Age-Related Macular Degeneration}

Age related Macular Degeneration (AMD) is the leading cause of blindness in the elderly, which is targeted at the RPE. With the progress of AMD, it can be developed into two different forms of advanced AMD: "dry" atrophic AMD, characterized in that the RPE aging and RPE loss and the "wet" neovascular AMD, characterized by choroidal vascular abnormal growth of RPE activation. DanHong (Mookherjee et al., 2010) found that BMP4 was differentially expressed in two types AMD. BMP4 is highly expressed in atrophic AMD. In contrast, the expression of RPE in BMP4 was low in neovascular AMD damage. Zhu et al. (2009a) in vitro study revealed more detailed mechanisms involved in BMP4 mediated oxidative stress in RPE cell senescence. Under oxidative stress, RPE cells increased the expression of BMP4 and further activated its downstream signaling pathway to induce P53 and P21Cip1/WAF1, but inhibited phosphorylation by Smad and P38 MAPK pathway in Rb cells. The increased P53 and decreased the phosphorylation of $\mathrm{Rb}$ triggers RPE cell senescence, the cell senescence of RPE BMP4 mediated by BMP4 antagonist or P38 inhibitor partially blocked, may inhibition of oxidative stress and aging and treatment of dry AMD. BMP4 is known to participate in the decision of individual AMD in the form of advanced molecular switch, Xu et al. (2011; Zhu et al., 2009b) research shows that when the expression of TNF is high (7 days postlaser), decreased the expression of BMP4 in $\mathrm{CNV}$ damage in mice induced by laser, while reducing the level of TNF (14 days postlaser), re expression BMP4 in RPE. The BMP4 promoter and the two specific protein (Sp1) targets of TNF were determined. We found the downregulation of BMP4 expression by TNF in CNV and the establishment of the mechanism are helpful to identify the new target for AMD therapy.

\section{Outlook}

The latest research shows that BMP4 is associated with a variety of eye diseases such as: BMP4 haploid deficiency may be (Xu et al., 2011) causes the clinical manifestation of Frias syndrome; BMP4 is retinal dystrophy and abnormal brain main gene (Martínez-Fernández et al., 2014); BMP4 may through the stimulation of VEGF from RPE cells in regulating the release and Diabetic Retinopathy (DR) play a role (Bakrania et al., 2008) eye associated with angiogenesis in. The treatment of the biological function of BMP4 in human congenital cleft palate, fracture, osteoporosis, bone defect, dental disease, congenital heart disease and pyloric stenosis and other diseases will have wide application prospect.

\section{Conclusion}

With the gradual maturation of the research methods and the increase of BMP4, the role of eye tissue gradually clear, with the occurrence of many eye diseases will also be elucidated.

\section{Acknowledgment}

Authors would like to thank the funding support from Jilin University Bethune support program 470110000520 and Jilin University outstanding young teacher training program 419080500586.

\section{Author's Contributions}

Liang Jiaming and Wang Qian: Contributed to the writing of the manuscript.

Liu Xiaohe: Participated in all paper searching.

Wang Shurong and Zhang Yan: Conceived and designed the research.

\section{Ethics}

All authors read and approved the final version and are responsible for any ethical issue that may arise after the publication of this manuscript.

\section{References}

Allison, P., D. Espiritu and T.D. Camenisch, 2016. BMP2 rescues deficient cell migration in Tgfbr $3^{-/}$epicardial cells and requires Src kinase. Cell Adh Migr., 10: 259-268. DOI: 10.1080/19336918.2015.1119362

Bakrania, P., M. Efthymiou, J.C. Klein, A. Salt and D.J. Bunyan et al., 2008. Mutations in BMP4 cause eye, brain and digit developmental anomalies: Overlap between the BMP4 and hedgehog signaling pathways. Am. J. Hum. Genet., 82: 304-319.

DOI: 10.1016/j.ajhg.2007.09.023 
Balenci, L., C. Wonders, B.L. Coles, L. Clarke and D. van der Kooy, 2013. Bone morphogenetic proteins and secreted frizzled related protein 2 maintain the quiescence of adult mammalian retinal stem cells. Stem Cells, 31: 2218-2230. DOI: 10.1002/stem.1470

Boswell, B.A. and L.S. Musil, 2015. Synergistic interaction between the fibroblast growth factor and bone morphogenetic protein signaling pathways in lens cells. Mol. Biol. Cell, 26: 2561-2572. DOI: $10.1091 / \mathrm{mbc} . \mathrm{E} 15-02-0117$

Huang, J., Y. Liu, A. Oltean and D.C. Beebe, 2015a. Bmp4 from the optic vesicle specifies murine retina formation. Dev. Biol., 402: 119-126. DOI: $10.1016 /$ j.ydbio.2015.03.006

Ito, Y.A. and M.A. Walter, 2014. Genomics and anterior segment dysgenesis: A review. Clin. Exp. Ophthalmol., 42: 13-24. DOI: 10.1111/ceo.12152

Jensen, A.M., 2005. Potential roles for BMP and Pax genes in the development of iris smooth muscle. Dev. $\quad$ Dynam., 232: 385-392. DOI: $10.1002 /$ dvdy.20224

Kallioniemi, A., 2012. Bone morphogenetic protein 4a fascinating regulator of cancer cell behavior. Cancer Genet., 205: 267-277. DOI: 10.1016/j.cancergen.2012.05.009

Kobayashi, T., K. Yasuda and M. Araki, 2010. Coordinated regulation of dorsal bone morphogenetic protein 4 and ventral Sonic hedgehog signaling specifies the dorso-ventral polarity in the optic vesicle and governs ocular morphogenesis through fibroblast growth factor 8 upregulation. Dev. Growth Differ., 52: 351-363. DOI: 10.1111/j.1440-169X.2010.01170.x

Lee, J.H., D.H. Bhang, A. Beede, T.L. Huang and B.R. Stripp et al., 2014. Lung stem cell differentiation in mice directed by endothelial cells via a BMP4-NFATc1-Thrombospondin-1 axis. Cell, 156: 440-455. DOI: 10.1016/j.cell.2013.12.039

Lynch, A.M., B.D. Wagner, N. Mandava, A.G. Palestine and P.M. Mourani et al., 2016. The relationship of novel plasma proteins in the early neonatal period with retinopathy of prematurity. Invest. Ophthalmol. Vis. Sci., 57: 5076-5082. DOI: 10.1167/iovs.16-19653

Macé, M., S.D. Galiacy, A. Erraud, J.E. Mejía and H. Etchevers et al., 2011. Comparative transcriptome and network biology analyses demonstrate antiproliferative and hyperapoptotic phenotypes in human keratoconus corneas. Invest. phthalmol. Vis. Sci., 52: 6181-691. DOI: 10.1167/iovs.10-70981

Mao, M., R.S. Smith, M.V. Alavi, J.K. Marchant and M. Cosma et al., 2015. Strain-dependent anterior segment dysgenesis and progression to glaucoma in Col4al mutant mice. Invest Ophthalmol. Vis. Sci., 56: 6823-6831. DOI: 10.1167/iovs.15-17527
Martínez-Fernández, M.L., E. Bermejo-Sánchez, B. Fernández, A. MacDonald and J. Fernández-Toral et al., 2014. Haploinsufficiency of BMP4 gene may be the underlying cause of Frías syndrome. Am. J. Med. Genet. A., 164A: 338-345. DOI: 10.1002/ajmg.a.36224

Maruyama, Y., S. Mikawa, Y. Hotta and K. Sato, 2006. BMP4 expression in the developing rat retina. Brain Res., 1122: 116-121. DOI: 10.1016/j.brainres.2006.08.130

Mookherjee, S., D. Banerjee, S. Chakraborty, A. Banerjee and I. Mukhopadhyay et al., 2010. Association of $I L 1 A$ and $I L 1 B$ loci with primary open angle glaucoma. BMC Med. Genet., 11: 99-99. DOI: $10.1186 / 1471-2350-11-99$

Müller, F., H. Rohrer and A. Vogel-Höpker, 2007. Bone morphogenetic proteins specify the retinal pigment epithelium in the chick embryo. Development, 134: 3483-3493. DOI: 10.1242/dev.02884

Napier, H.R. and S.H. Kidson, 2007. Molecular events in early development of the ciliary body: A question of folding. Exp. Eye Res., 84: 615-625. DOI: 10.1016/j.exer.2006.07.012

Pereira da Silva, L., B. Miguel Neves, L. Moura, M.T. Cruz and E. Carvalho, 2014. Neurotensin decreases the proinflammatory status of human skin fibroblasts and increases epidermal growth factor expression. Int. J. Inflam. DOI: 10.1155/2014/248240

Rubinstein, T.J., A.C. Weber and E.I. Traboulsi, 2016. Molecular biology and genetics of embryonic eyelid development. Ophthalmic Genet., 11: 1-8. DOI: $10.3109 / 13816810.2015 .1071409$

Sandri, G., M.C. Bonferoni, S. Rossi, A. Delfino and F. Riva et al., 2016. Platelet lysate and chondroitin sulfate loaded contact lenses to heal corneal lesions. Int. J. Pharm., 25: 188-196. DOI: 10.1016/j.ijpharm.2016.05.045

Ueno, M., K. Asada, M. Toda, U. Schlötzer-Schrehardt and K. Nagata et al., 2016. Gene signature-based development of ELISA assays for reproducible qualification of cultured human corneal endothelial cells. Invest Ophthalmol. Vis. Sci., 57: 4295-305. DOI: $10.1167 /$ iovs. 16-19806

Xu, J., D. Zhu, S. He, C. Spee and S.J. Ryan et al., 2011. Transcriptional regulation of bone morphogenetic protein 4 by tumor necrosis factor and its relationship with age-related macular degeneration. FASEB J., 25: 2221-2233. DOI: 10.1096/fj.10-178350

Yao, H., H. Li, S. Yang, M. Li and C. Zhao et al., 2016. Inhibitory effect of bone morphogenetic protein 4 in retinal pigment epithelialmesenchymal transition. Sci. Rep., 6: 32182-32182. DOI: $10.1038 /$ srep32182 
Zhang, R., H. Huang, P. Cao, Z. Wang and Y. Chen et al., 2013. Sma- and Mad-related protein $7(\operatorname{Smad} 7)$ is required for embryonic eye development in the mouse. J. Biol. Chem., 288: 10275-1085.

DOI: $10.1074 /$ jbc.M112.416719

Zhang, Y., L.K. Yeh, S. Zhang, M. Call and Y. Yuan et al., 2015. Wnt/ $\beta$-catenin signaling modulates corneal epithelium stratification via inhibition of Bmp4 during mouse development. Development, 142: 3383-3393. DOI: 10.1242/dev.125393
Zhu, D., X. Deng, J. Xu and D.R. Hinton, 2009a. What determines the switch between atrophic and neovascular forms ofage related macular degeneration? - The role of BMP4 induced senescence. Aging, 1: 740-745. DOI: 10.18632/aging. 100078

Zhu, D., J. Wu, C. Spee, S.J. Ryan and D.R. Hinton, 2009b. BMP4 mediates oxidative stress-induced retinal pigment epithelial cell senescence and is overexpressed in age-related macular degeneration. J. Biol. Chem., 284: 9529-9539. DOI: 10.1074/jbc.M809393200 